\title{
Active Region Loop Models and Observations
}

\author{
E. Landi \\ Artep, Inc., Ellicott City, MD 21042 and Naval Research Laboratory, \\ Washington, DC 20375, USA \\ M. Landini \\ University of Florence, Largo E.Fermi 2, 51025 Firenze, Italy
}

\begin{abstract}
In the present work we compare CDS observations of active region loops and predictions from a steady-state theoretical model developed assuming different functional forms of steady-state loop heating. The present work shows that in no case agreement with observations is achieved, that filamentation is present, and that despite its limitation, CDS is a precious tool for loop physics.
\end{abstract}

\section{Introduction}

Results from imaging instruments working at X-ray, UV and EUV wavelengths have shown that plasma loops are a fundamental component for the quiet and active solar corona. Their understanding is an essential step required for the knowledge of the structure of the solar atmosphere, as well as of the mechanism(s) that heat the solar corona. However, recent analyses of coronal observations provide contradictory results on coronal heating, and provide no definitive answer to the problem of whether loops are monolithic or filamentary structures.

In the present work we compare CDS observations with a steady-state, monolithic loop model. CDS observations consist of spatially resolved spectra in the EUV region at various temperatures, which allow us to measure the loop density, temperature, emission measure, filling factor, and mass motions as a function of position along the loop. Such quantities can be compared with the loop temperature and density profile predicted by the model. The comparison is carried out with models calculated assuming different functional forms of the loop heating, assumed to be stationary, so that it is possible to discriminate among the proposed heating scenarios.

The present work also shows that even with its moderate spatial resolution, CDS can provide important constraints to theoretical models, thanks to its diagnostic capabilities, superior to those imagers with higher resolution.

\section{Model}

The model consists of a one-dimensional, single fluid model that solves the equations of conservation of mass, energy and momentum, first developed by Landini 
\& Monsignori Fossi (1981). The problem is solved under the assumptions of 1) steady state, 2) constant cross section. The model input parameters, chosen by the user, are the loop total length (measured from CDS imaged spectra), loop pressure at the footpoint (measured from CDS observations), conductive flux and velocity at the footpoints. The model provides the main physical quantities at all positions along the loop, that can be compared with observations. A complete description of the model can be found in Landi \& Landini (2004).

In the present work, we will concentrate in comparing the predicted density and temperature profiles with observations. Also, the assumption of monolithic loop can be independently tested by using CDS measurements of the emission measure and electron density, coupled with the loop cross section width as observed by CDS monochromatic images. These quantities allow for the measurement of the loop filling factor: in case the latter quantity is smaller than one, the loop is most likely composed by a number of unresolved strands.

\section{Observations}

The observations were recorded on April 29th 1997 with CDS (Harrison et al. 1995), using also magnetograms from MDI and images from EIT and Yohkoh to help in the loop shape selection. CDS scanned an active region on the solar disc centred at around $\left(-100^{\prime \prime},-400^{\prime \prime}\right)$, with a field of view of $243.6^{\prime \prime} \times 240.2^{\prime \prime}$ and an exposure time of $60 \mathrm{~s}$ for each slit position. Only lines from selected ions were telemetred to ground: O III,V, Ne V, Mg VII,VIII,IX, Si XI,XII, Fe XII,XIII,XIV,XVI. Electron density can be measured from Fe XIII line ratios, and the electron temperature from line ratios from different $\mathrm{Fe}$ ions and from an emission measure analysis.

CDS raw data were cleaned and calibrated, and cosmic rays removed using the standard CDS software. The selection of the loop shape was made using monochromatic images from all CDS lines, and broad-band images from EIT and Yohkoh. The selected loop shape is shown in Figure 1. The loop shape was divided in 21 subsections, each analyzed separately.

The background to be subtracted from each of the subsections has been determined by averaging the emission of the surrounding pixels closest to each of them: in this way its own background has been associated to each loop subsection. This choice has allowed us to take into account the variation of the background emission along the loop shape, so that it could be accurately removed from the loop signal. Such subtraction is of crucial importance to this study. This subtraction has shown that only lines from Si XI,XII, Fe XII,XIII,XIV,XVI have a component emitted by the loop and not only by the background. Plasma diagnostics has been carried out using the CHIANTI database (Dere et al. 1997).

\section{Results}

Plasma diagnostics: The electron temperature, density and pressure along the loop are shown in Figure 2. The electron temperature has been measured using two different methods: line intensity ratios between different Fe ions (dashed lines, each corresponding to a different ion pair) and an emission measure analysis (individual points). The excellent agreement between the two different 
Fe XVI $360 \mathrm{~A}+$ EIT 171 composite
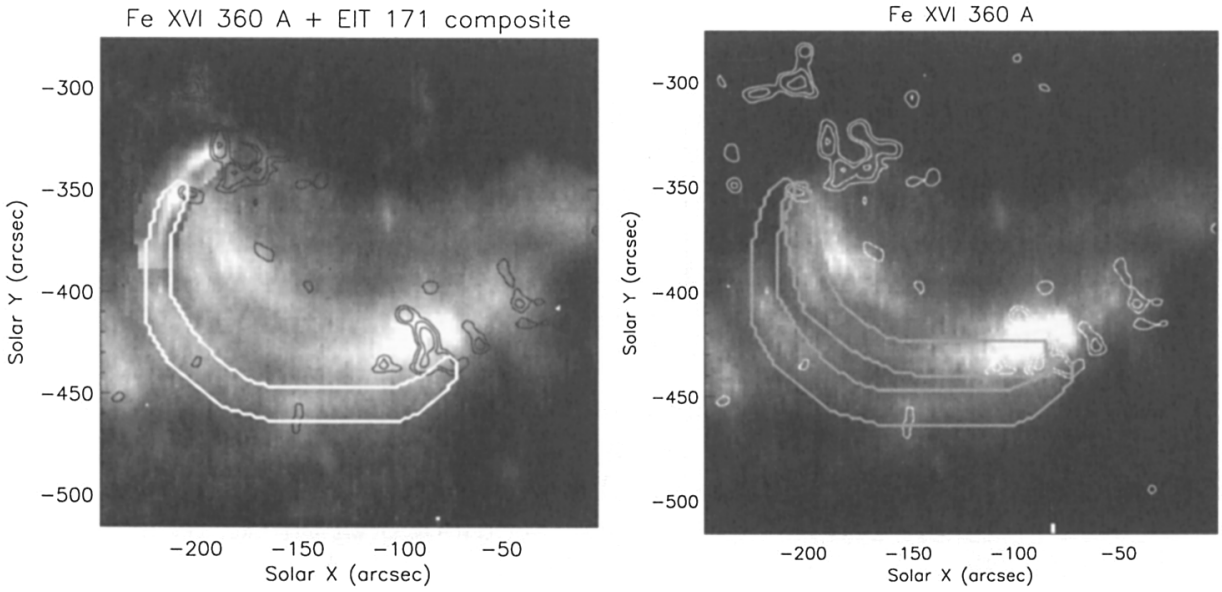

Figure 1. CDS field of view and selected loop shape. The outermost loop has been used for the present analysis.
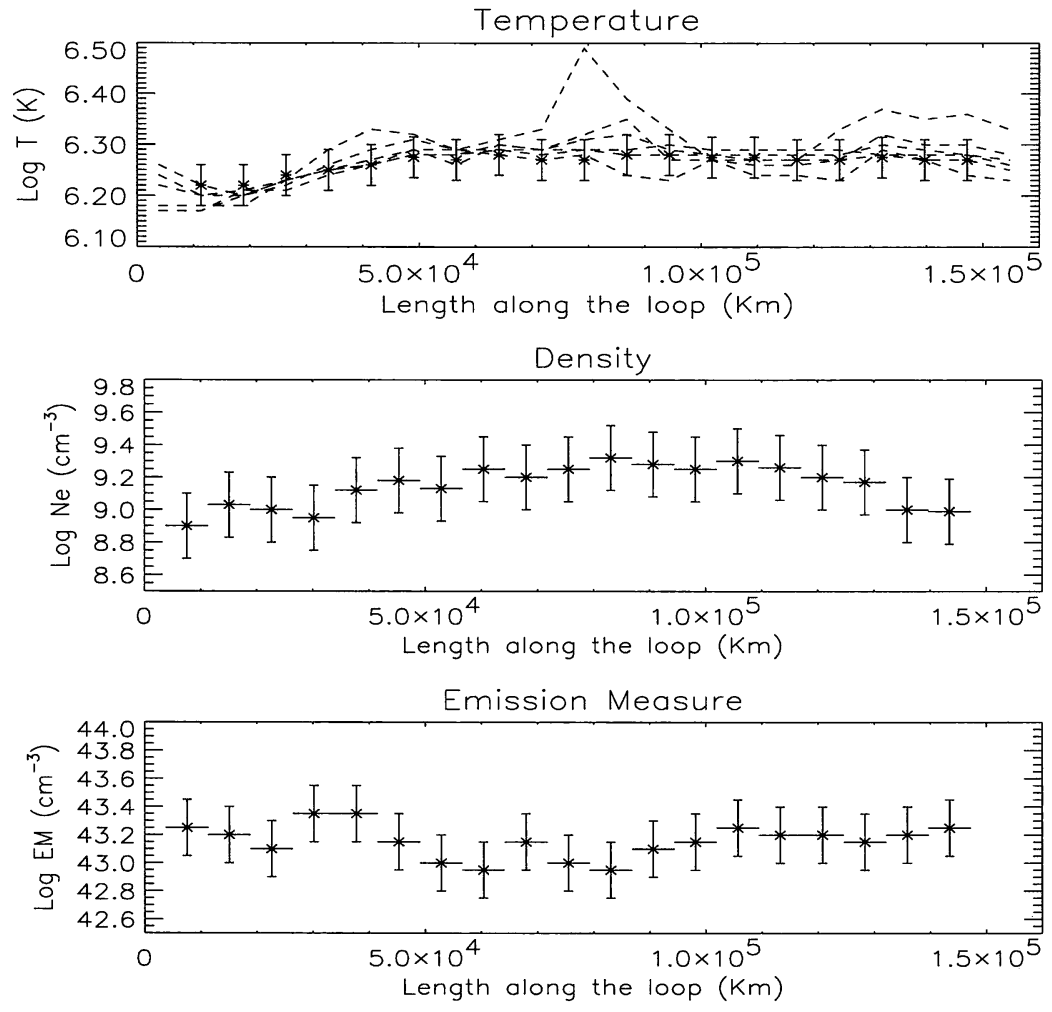

Figure 2. Plasma diagnostics as a function of position along the loop, from CDS line ratios. The two footpoints have not been displayed because their plasma is multithermal and the diagnostic is inaccurate. 

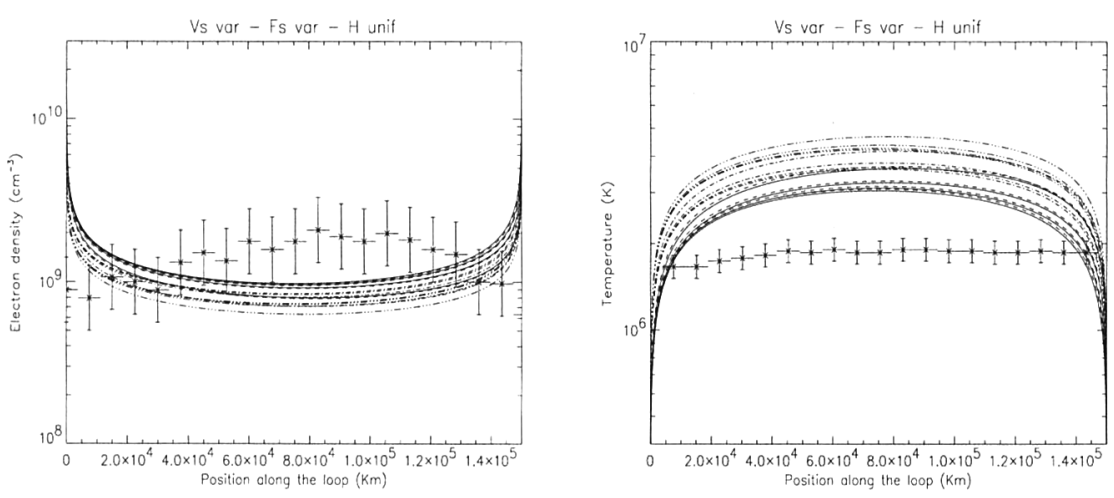

Figure 3. Comparison between CDS plasma diagnostics and predictions based on uniform heating. Left: temperature; Right: electron density.
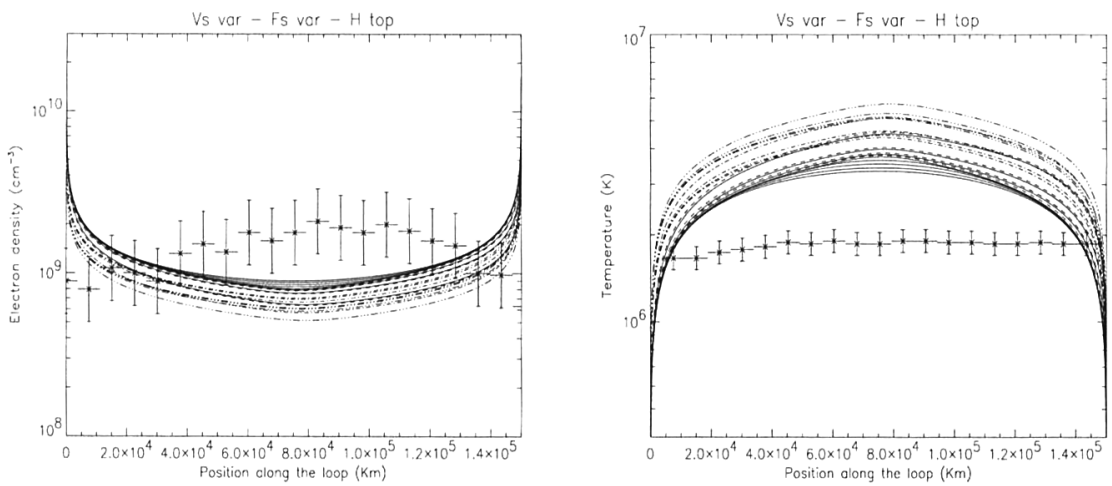

Figure 4. Comparison between CDS plasma diagnostics and predictions based on heating at the top. Left: temperature; Right: electron density.
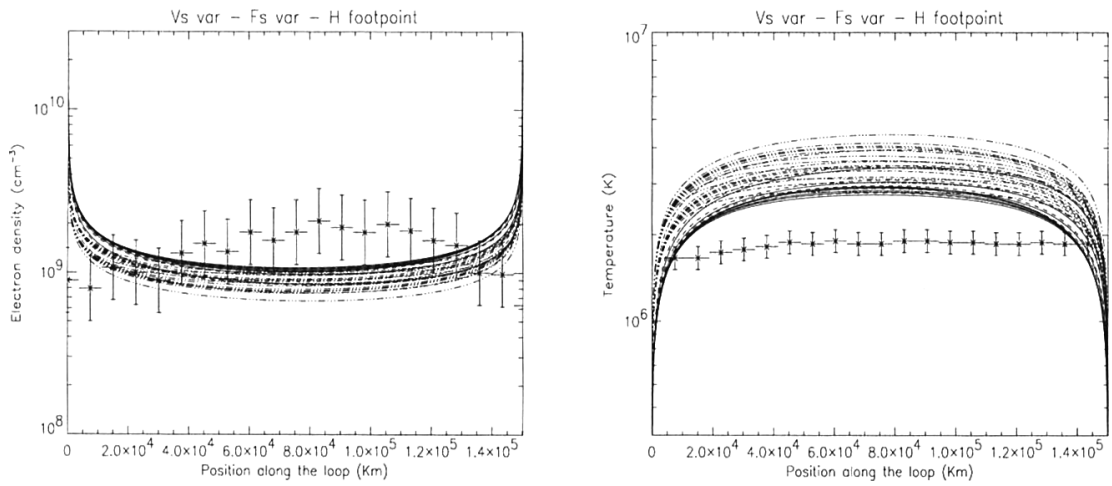

Figure 5. Comparison between CDS plasma diagnostics and predictions based on heating at the footpoint. Left: temperature; Right: electron density. 
methods, and among the line intensity ratios, shows that the plasma is isothermal and that the background subtraction has been accurate. The temperature profile is flat along all the loop length; only at the footpoints (not shown in Figure 1) the loop plasma is not isothermal, with temperatures ranging from $10^{4}$ to $3 \times 10^{6} \mathrm{~K}$. The electron density, pressure and emission measure are approximately constant; since the loop cross-section is also uniform, this means that the filling factor is also constant, with a value in the $10^{-2}-10^{-1}$ range, showing that the loop is composed by unresolved strands.

Comparison with models: The comparison between observed and predicted temperature and density profiles is shown in Figures 3,4 and 5 for three different heating functions: uniform, concentrated at the loop top, and concentrated at the loop footpoint respectively. In each case, we have varied the conductive flux at the footpoint $F_{0}$ assuming $F_{0}=0,-10^{6},-5 \times 10^{6}$ and $-10^{7} \mathrm{erg} \mathrm{cm}^{-2} \mathrm{~s}^{-1}$, and the footpoint velocity $v_{0}$ assuming $v_{0}=0.9,2.7$ and $9 \mathrm{~m} \mathrm{~s}^{-1}$. Figures 3 to 5 show that while the predicted plasma density is in all cases of the same order of the observations, the temperature is always hotter than observed, and its profile is not enough uniform to match the CDS results.

The heating function profile that most closely resembles the observed one is the one with footpoint heating and a strong conductive flux at the footpoint. However, it still does not reproduce the observations.

\section{Conclusions}

The present work shows that 1) CDS observations can provide precious contributions to loop physics even with its moderate spatial resolution; 2) the loop filling factor is smaller than unity, so that filamentation must be present, as suggested by broad band imagers; 3) a steady-state heating function is not able to reproduce the observations, whatever spatial distribution is assumed.

Therefore, it is necessary to build models that take into account impulsive heating, non-equilibrium effects on loop plasma due to impulsive heating, and filamentation.

Acknowledgments. The work of E. Landi is supported by the NASA Living with a Star program.

\section{References}

Dere K.P., Landi E., Mason H.E., Monsignori Fossi B.C., \& Young P.R. 1997, A\&AS, 125,149

Harrison, R.A., Sawyer, E.C., Carter, M.K., et al. 1995, Solar Physics, 162, 233 Landi, E., \& Landini, M. 2004, ApJ, submitted

Landini, M., \& Monsignori Fossi, B.C. 1981, A\&A, 102, 391 

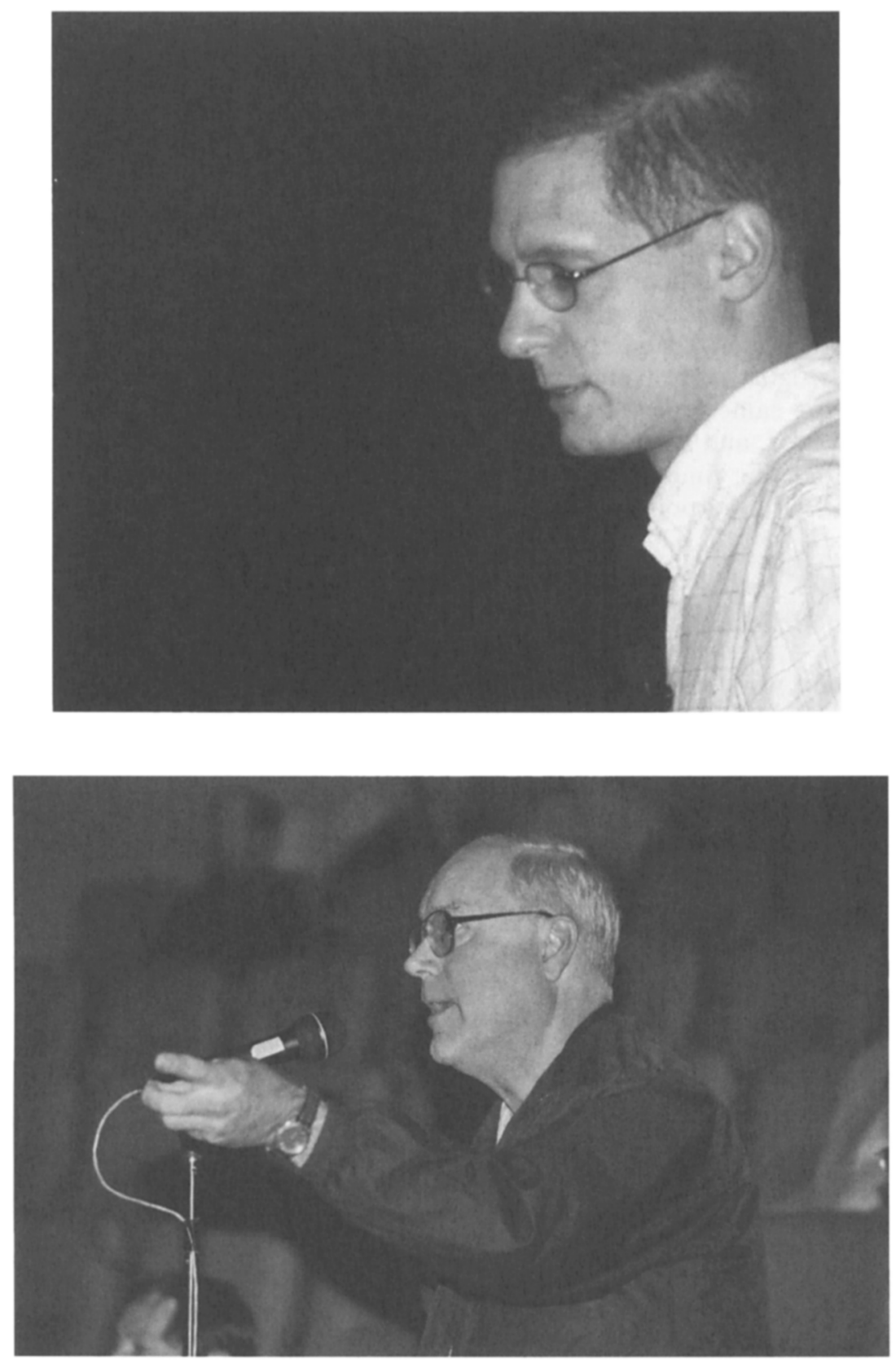